\title{
Design of a new scheme for multi-hop wireless networks using decode-and-forward strategy
}

\author{
Iker Alustiza*, Mikel Hernaez and Pedro Crespo
}

\begin{abstract}
This paper proposes a Decode-and-Forward (DF) relaying scheme for the multi-hop transmission in wireless networks, where the information generated by an independent source has to be sent to a far destination based on multiple-relay cooperation. The proposed DF scheme blends together convolutional channel coding with linear combination of blocks of data over a finite field using very short block lengths $(K=13)$. We provide an extrinsic information transfer (EXIT) chart analysis to understand the good performance behavior of the proposed scheme when compared with other referenced schemes using much larger block lengths. This fact is corroborated by a set of Monte Carlo simulations. Moreover, the proposed DF scheme is suitable for large multi-hop networks since a negligible performance degradation is obtained when adding more hops.
\end{abstract}

Keywords: Multihop transmission; Wireless networks; Decode-and-forward; EXIT charts

\section{Introduction}

In a multihop network, the data transmission between the source and the corresponding destination is realized with the aid of intermediates nodes (relays). These networks have been the focus of an intense research in the recent years, where Multihop Communication has been shown to be an effective method for establishing connectivity between nodes of a network where direct transmission is not feasible due to coverage or battery life issues [1-5].

In [1-3] and reference therein, several theoretical aspects of multihop network were presented. Concretely, in [1], efficient routing protocols for several power allocations in fading were presented. Based on these protocols, the authors in [4] proposed a Decode-and-Forward (DF) relaying strategy based on combining together lowdensity parity-check (LDPC) codes with a linear combination of blocks of data over a finite field. It was showed that for blocks of $K=1,500$ information bits, the proposed scheme outperformed two reference schemes based on the previous works addressing similar problems $[5,6]$.

In the current manuscript, we propose a DF relaying strategy which uses a terminated convolutional code and an improved version of the non-binary block-wise linear

*Correspondence: ikeralustiza@gmail.com

Centro De Estudios e Investigaciones Técnicas de Gipuzkoa (CEIT) and TECNUN (University of Navarra), 20008 Donostia-San Sebastián, Spain combination of [4]. At each node, the decoding scheme is based on applying the sum-product algorithm (SPA) to a factor graph [7] describing the $a$ posteriori probability of the communication scenario at the corresponding node. By using blocks of only $K=13$ information bits, the performance of the proposed scheme outperforms the scheme of [4] (and consequently, [5,6]) where 1,500 bits were used, making the proposed scheme particularly attractive for low-latency applications.

This paper, extending the work carried out in [8], provides an extrinsic information transfer (EXIT) chart analysis [9] to understand the good performance behavior of the proposed scheme based on short length codes. Although EXIT charts provide good BER convergence predictions only for long-length codewords (which is not our case), it gives us a good insight on why the proposed system outperforms the LDPC-based system. Moreover, this same tool has been previously used with short-length terminated convolutional codes by the authors in [10] with successful results. For a more accurate prediction of the BER convergence for short length codes, we refer to [11], where a method for computing lower bounds based on a EXIT band chart is proposed.

The rest of the manuscript is organized as follows: Section 2 introduces the network model, whereas the proposed DF scheme is presented in Section 3. In Section 4, an analysis of the code based on EXIT charts is performed.

\section{是 Springer}

(c) 2015 Alustiza et al. This is an Open Access article distributed under the terms of the Creative Commons Attribution License (http://creativecommons.org/licenses/by/4.0), which permits unrestricted use, distribution, and reproduction in any medium, provided the original work is properly credited. 
Section 5 discusses the obtained Monte Carlo simulations results, and finally, Section 6 concludes the manuscript.

\section{Network model}

We study the problem where a user wants to transmit data to a destination through a mutihop wireless network. For the sake of simplicity, we have imposed a straightline geometry and unity distance between two consecutive nodes, so we can easily compute distances and signal attenuations between nodes. In order to avoid possible interferences in the network, we design the system in time division multiple access (TDMA) mode.

The nodes are assumed to work in half-duplex mode; hence, a node can either receive or transmit data, but not both at the same time. Therefore, the data is transmitted in a progressive way through the simplified linear network. That is, one node can listen to all the previous nodes but not to the following ones. Due to the wireless nature of the signals, we consider Rayleigh fading and path loss attenuation. Finally, due to the wireless environment and following $[4,5]$, we do not constrain the transmission range of each node to one hop, as done in [1-3].

Let $\mathfrak{M}=\{s, 1, \ldots, m-1, m\}$ denote the set of nodes in the network, where $s$ and $m$ are the source and destination nodes, respectively. We define $R=\{1, \ldots, m-1\} \subset \mathfrak{M}$ as the subset formed by the intermediate nodes (relays), and $T_{j}=\{s, 1, \ldots, j-1\} \subset \mathfrak{M}$ (for $j=1, \ldots, m$ ) as the subset of nodes from which node $j$ receives their transmitted symbols. Figure 1 depicts the network model.

Regarding the links between nodes, we consider independent (orthogonal) quasi-static Rayleigh flat-fading channels and channel state information at the receivers (coherent detection), where the $t$-sampled baseband (complex) link input-output $(S-Y)$ relation from node $i$ to node $j$ at time $t$ is given by

$$
Y_{t}=\left|\Lambda^{i j}\right| \sqrt{d_{i j}^{-\delta}} S_{t}+N_{t}^{i j}, \quad S_{t} \in \mathbb{C}
$$

with $d^{-\delta}$ modeling the path loss attenuation, where $d$ is the number of hops (units) between the transmitting and the receiving node and $\delta$ is the attenuation exponent. The fading coefficient $\Lambda$ and the additive Gaussian noise $N_{t}$ are circularly symmetric complex Gaussian random variables of zero mean and variance one and $N_{0}$, respectively.
A realization of $\Lambda$ is assumed to remain constant for the duration of the transmitted block.

\section{Proposed DF scheme}

Like most of the DF schemes, the proposed scheme can be partitioned into two blocks: the decode block, where the information sequence is estimated; and the forward block, where the estimated sequence is encoded and forwarded. In this case, the forward part at the source node is implemented in a different manner than the ones at the relay nodes; therefore, it is explained first.

We consider an i.i.d binary source that generates information blocks $\mathbf{U} \in\{0,1\}^{K}$. Then, the information sequence is encoded by a rate $R=K / N$ convolutional code. The resulting encoded binary codeword $\mathbf{C} \triangleq$ $\left\{C_{n}\right\}_{n=1}^{N} \in\{0,1\}^{N}$ is interleaved by a random interleaver $\Pi$ yielding the interleaved sequence $\mathbf{X}$ with $X_{n}=C_{\Pi(n)}$. Finally, $\mathbf{X}$ is mapped into the sequence of symbols $\mathbf{S} \in S^{M}$ chosen from a $2^{q}$-ary complex signal constellation $S \subset \mathbb{C}$ according to the bit-to-symbol mapping $\mu:\{0,1\}^{q} \rightarrow S$ (e.g., Gray mapping), thus, $M=N / q$.

Since we next present the operations performed at each relay node, we will intentionally omit any references to the particular node $j$. However, when considering the entire network, we will use the upper index $j$ to discern between different nodes.

\subsection{Forward}

At each node $j \in R$, the decode block, which is explained later, outputs the estimated information sequence $\widehat{\mathbf{U}}$, which is then encoded and interleaved using the aforementioned convolutional code and interleaver. The resulting binary sequence $\mathbf{X}$ is partitioned into the $q$-length sub-sequences $\mathbf{X}_{l} \triangleq\left\{X_{l q+i}\right\}_{i=1}^{q}$, where $l=0, \ldots, M-1$. Let $\phi: \mathbb{F}_{2}^{q} \rightarrow \mathbb{F}_{2^{q}}$ denote the one-to-one mapping from $q$-length binary sequence ${ }^{1}$ into elements of $\mathbb{F}_{2 q}$. Thus, the non-binary symbols $\left\{V_{l}\right\}_{l=0}^{M-1} \in \mathbb{F}_{2^{q}}^{M}$ are computed from each sub-sequence as $V_{l}=\phi\left(\mathbf{X}_{l}\right)$.

Let us now denote $\bar{l} \triangleq|M / 2-l|$ and consider the set of pairs $\left\{\left(V_{l}, V_{\bar{l}}\right): l=0, \ldots, M-1\right\}$. By symmetry, we have that $\left\{\left(V_{l}, V_{\bar{l}}\right)\right\}_{l=0}^{M / 2-1}=\left\{\left(V_{l}, V_{\bar{l}}\right)\right\}_{l=M / 2}^{M-1}$. Thus, at the relays, we only consider $l=0, \ldots, M / 2-1$. Each pair $\left(V_{l}, V_{\bar{l}}\right)$ is linearly combined over the field $\mathbb{F}_{2 q}$ and mapped into signal points of the $2^{q}$-ary constellation $S$. That is, for $t=1, \ldots, M / 2$ and $l=t-1$

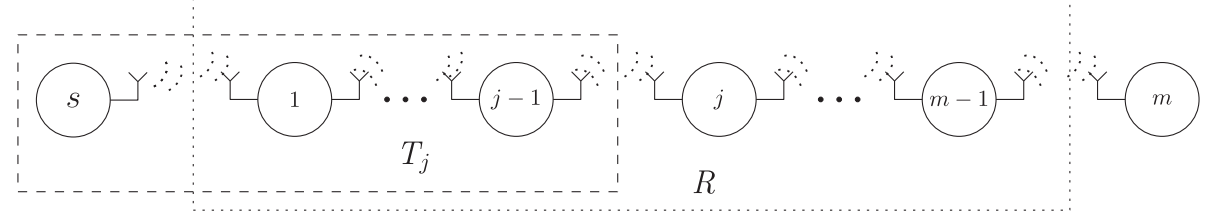

Figure 1 Network model. 


$$
S_{t}=\mu_{\mathbb{F}_{2 q}}\left(h_{1} V_{l}+h_{2} V_{\bar{l}}\right) \in \mathbb{F}_{2}^{q}
$$

where $\mu_{\mathbb{F}_{2} q}(x) \triangleq \mu\left(\phi^{-1}(x)\right) \forall x \in \mathbb{F}_{2 q}$. The network vector coefficients $\mathbf{h}=\left(h_{1}, h_{2}\right)$, with $\mathbf{h} \in \mathbb{F}_{2 q}^{2}$, are fixed by the network so that all the vector coefficients in the network are linearly independent. We further constrain the election to $\left\{h_{i}\right\}_{i=1,2} \neq 0$. Finally, note that the length, $N / 2$, of the sequences transmitted by the relays is half of the length, $N$, of the sequences transmitted by the source. Figure 2 provides a graphical explanation of the encoding process.

\subsection{Decode}

Let us consider an arbitrary node $j \in R \cup m$ that receives the sequences sent by the nodes $i \in T_{j}$ (i.e., all the nodes located at its left-hand side, see Figure 1). This node has $\left|T_{j}\right|$ incoming links with received sequences $\left(\mathbf{y}^{1}, \ldots, \mathbf{y}^{\left|T_{j}\right|}\right)$. For the sake of clarity, through this sub-section, we define $\mathbf{y} \triangleq\left(\mathbf{y}^{1}, \ldots, \mathbf{y}^{\left|T_{j}\right|}\right)$ and denote $\mathbf{y}_{t}$ as the $\left|T_{j}\right|$ symbols received at time instant $t$, which are related to the elements $V_{l}$ and $V_{\bar{l}}$ (see (1) and (2)).

The aim of the decoder is to implement a bitwise MAP decoding. That is, to find the values $\left\{u_{k}\right\}_{k=1}^{K}$ that are most likely given $\underline{y}$, i.e.,

$$
\hat{u}_{k}=\arg \max _{u_{k}} \sum_{\sim u_{k}} P_{\mathbf{U} \mid \underline{\mathbf{Y}}}(\mathbf{u} \mid \underline{\mathbf{y}})
$$

where $\sim u_{k} \triangleq\left\{u_{k^{\prime}}\right\}_{k^{\prime} \neq k}$. Since a convolutional code is used, the SPA algorithm reduces to (BCJR algorithm, see Figure 3)

$\hat{u}_{k}=\arg \max _{u_{k}} \sum_{\sim u_{k}} T_{k}\left(s_{k}, u_{k}, \mathbf{c}_{k}, s_{k+1}\right) \alpha\left(s_{k}\right) \beta\left(s_{k+1}\right) \cdot \prod_{n: c_{n} \in \mathbf{c}_{k}} \gamma\left(c_{n}\right)$,

where $\alpha$ and $\beta$, computed as

$$
\alpha\left(s_{k+1}\right)=\sum_{\sim s_{k+1}} T_{k}\left(s_{k}, u_{k}, \mathbf{c}_{k}, s_{k+1}\right) \alpha\left(s_{k}\right) \gamma\left(\mathbf{c}_{n}\right)
$$

and

$$
\beta\left(s_{k}\right)=\sum_{\sim s_{k}} T_{k}\left(s_{k}, u_{k}, \mathbf{c}_{k}, s_{k+1}\right) \beta\left(s_{k+1}\right) \gamma\left(\mathbf{c}_{n}\right),
$$

are the forward and backward messages passed from the adjacent state nodes to the factor node $T_{k}$ given by the Trellis of the convolutional code. Notice that in our case, the initial value $\alpha$ and $\beta$ is known (assuming that the convolutional code is terminated). The local functions $T_{k}\left(s_{k}, u_{k}, \mathbf{c}_{k}, s_{k+1}\right)$ describe the transitions allowed in the Trellis, indicating which combination of variables in its argument is valid. Moreover, $\mathbf{c}_{k}$ are the $N / K$ coded bits $\left\{c_{n}\right\}$ associated to $u_{k}$ and $\gamma$ are the the channel likelihoods of the coded bits. Due to the proposed forward block, these likelihoods are related to the interleaved binary symbols $x_{l q+i}$ through the $\left|T_{j}\right|$ incoming links, i.e., $\gamma\left(c_{n}\right)=$ $\gamma\left(x_{\Pi^{-1}(l q+i)}\right)$, where $\gamma\left(x_{l q+i}\right) \propto p\left(\underline{\mathbf{y}} \mid x_{l q+i}\right)$.

Since the channel is memoryless and assuming that the interleaved sequence is i.i.d., this probability can be factorized as

$$
p\left(\underline{\mathbf{y}} \mid x_{l q+i}\right) \propto \sum_{\sim x_{l q+i}, v_{l}} p\left(\mathbf{y}_{t} \mid v_{l}\right) \mathbb{1}\left[v_{l}=\phi\left(\mathbf{x}_{l}\right)\right] \prod_{i^{\prime} \neq i} P\left(x_{l q+i^{\prime}}\right)
$$

where

$$
p\left(\mathbf{y}_{t} \mid v_{l}\right)=\sum_{v_{\bar{l}}} p\left(\mathbf{y}_{t} \mid v_{l}, v_{\bar{l}}\right) P\left(v_{\bar{l}}\right)
$$

with

$$
P\left(v_{\bar{l}}\right)=\sum_{\mathbf{x}_{\bar{l}}} \mathbb{1}\left[v_{\bar{l}}=\phi\left(\mathbf{x}_{\bar{l}}\right)\right] \prod_{i=1}^{q} P\left(x_{\bar{l} q+i}\right),
$$

and $\mathbb{1}[\cdot]$ is an indicator function taking value 1 if its argument is true and 0 otherwise.

By using (2) and since $\Lambda$ and $d^{-\delta}$ are known at the receivers, the conditional probability density $p\left(\mathbf{y}_{t} \mid v_{l}, v_{\bar{l}}\right)$ can be written as

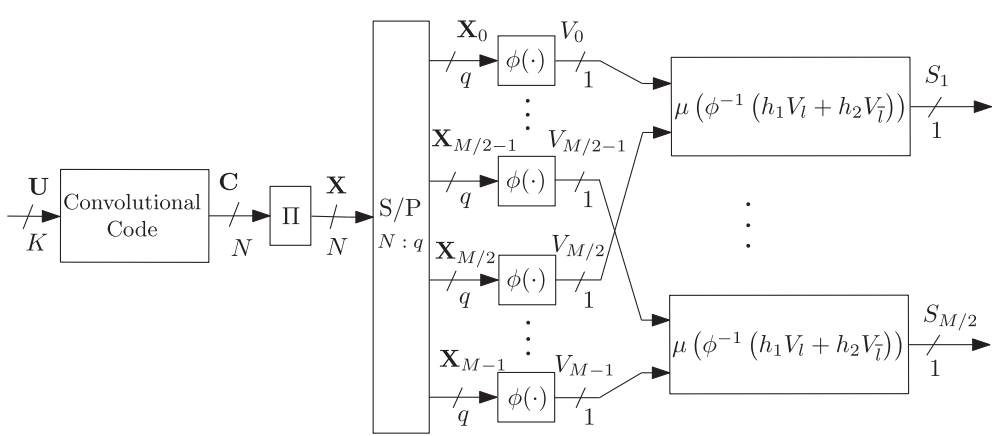

Figure 2 Encoder associated to the proposed code. 


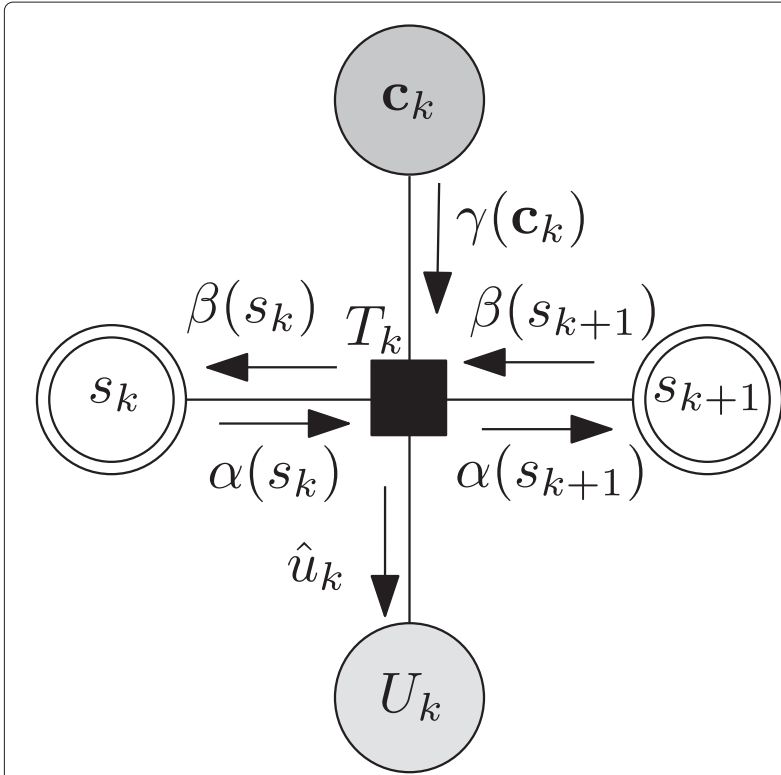

Figure $3 \mathrm{BCJR}$ algorithm for the convolutional code part of the decoding process.

$p\left(\mathbf{y}_{t} \mid v_{l}, v_{l}\right) \propto \exp \left\{\frac{-\sum_{i=1}^{\left|T_{j}\right|}\left\|y_{t}^{i}-\left|\Lambda^{i j}\right| \sqrt{d_{i j}^{-\delta}} \mu_{\mathbb{F}_{2 q}}\left(h_{1}^{i} v_{l}+h_{2}^{i} v_{l}\right)\right\|^{2}}{N_{0}}\right\}$,

where $\|\cdot\|$ denotes the euclidian norm.

Therefore, the marginalization of (3) can be efficiently performed by the SPA applied to the factor graph (Figure 4) derived from the factorization of $P_{\mathbf{U} \mid \underline{Y}}(\mathbf{u} \mid \mathbf{y})$ given by (4)-(9).

\section{Analysis of the code based on EXIT charts}

As it is shown in Figure 4, the factor graph of the decoder used by the SPA algorithm can be split in two blocks:

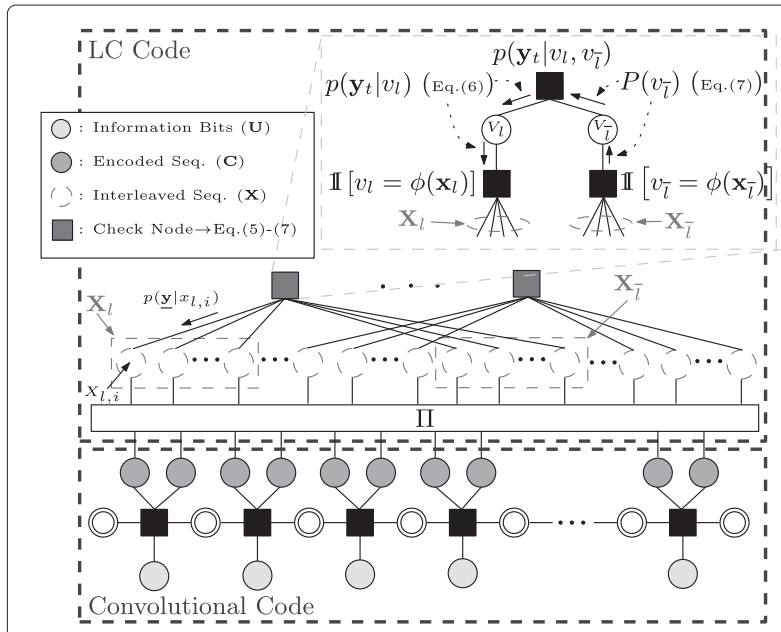

Figure 4 Factor graph employed for the decoding.
The linear combination (LC) block and the convolutional code block. Due to the iterative nature of this decoding algorithm, EXIT charts are a good method for visually understanding the behavior of the proposed scheme [9]. Given a code, the EXIT function associated is defined by the relation between the a priori mutual information at the input of the decoder (commonly denoted as $I_{a}$ ) and the corresponding extrinsic mutual information $I_{e}$ at its output, i.e., $I_{e}=T\left(I_{a}\right)$.

Given $q$ and $\mathbf{h}$, we denote the transfer function of the LC decoder as $I_{e}^{\mathrm{LC}}=T_{\mathbf{h}}^{q}\left(I_{a}^{\mathrm{LC}}\right)$, i.e., the extrinsic mutual information at the output of the LC decoder. Notice that, $T_{\mathbf{h}}^{q}(0)$ and $T_{\mathbf{h}}^{q}(1)$ represent the extrinsic information at the output of the LC decoder with no a priori and full a priori information on the information bits, respectively. As the mutual information at the input of the channel decoder $I_{a}^{\mathrm{CC}}$ is equal to $I_{e}^{\mathrm{LC}}$, the extrinsic mutual information at the output of the convolutional decoder is a function of $I_{e}^{\mathrm{LC}}$, that is, $I_{e}^{\mathrm{CC}}=T^{\mathrm{CC}}\left(I_{e}^{\mathrm{LC}}\right)$. Thus, for a successful decoding procedure, there must be an open gap between both EXIT curves so that the iterative decoding can proceed from $I_{e}^{\mathrm{CC}}=0$ to $I_{e}^{\mathrm{CC}}=1$. Otherwise, when both transfer functions cross, the iterative process will stop at a given extrinsic mutual information of the source bits $I_{e}^{\mathrm{CC}}<1$.

Figure 5a plots the EXIT charts for the LC code and for an $R=1 / 2$ convolutional channel code, for different network coefficients $\mathbf{h}=\left(h_{1}, h_{2}\right)$ and $q=4$. For the sake of clarity, we focus the analysis on the first node and, following the study in [12], it is also assumed an AWGN channel (i.e., $\Lambda^{s, 1}=1$ ) between this node and the source. Nevertheless, as it is shown in the EXIT chart study of [12], the same insight is obtained when assuming fading channels between nodes.

To compare the performance of our scheme with the one implemented in [4], Figure 5b plots the EXIT chart of an LDPC code of rate $R=0.416$. Notice, however, that a LDPC code is composed of several subgraphs, thus, several transfer functions (one for each compounding subgraph) are obtained and a direct representation of these functions will result in an $\mathrm{N}$-dimensional EXIT chart. By converting the $\mathrm{N}$-dimensional EXIT chart into a twodimensional EXIT chart, for example, by using the EXIT Chart Projection Algorithm proposed in [13], the same analysis could still be applied.

When using the convolutional code, one can observe from Figure 5a that the gap between the LC code and channel code curves is open for most of the network coefficients. On the contrary, in the LDPC code case, (Figure $5 \mathrm{~b}$ ) shows that this gap is close for all of the $\left(h_{1}, h_{2}\right)$ coefficients combinations. Therefore, the iterative decoding process will stop before reaching $I_{e}^{\mathrm{LDPC}}=1$. Both EXIT charts in Figure 5 are calculated with the same $a$ priori mutual information $I_{a}^{\mathrm{LC}}$, in other words, using the same 


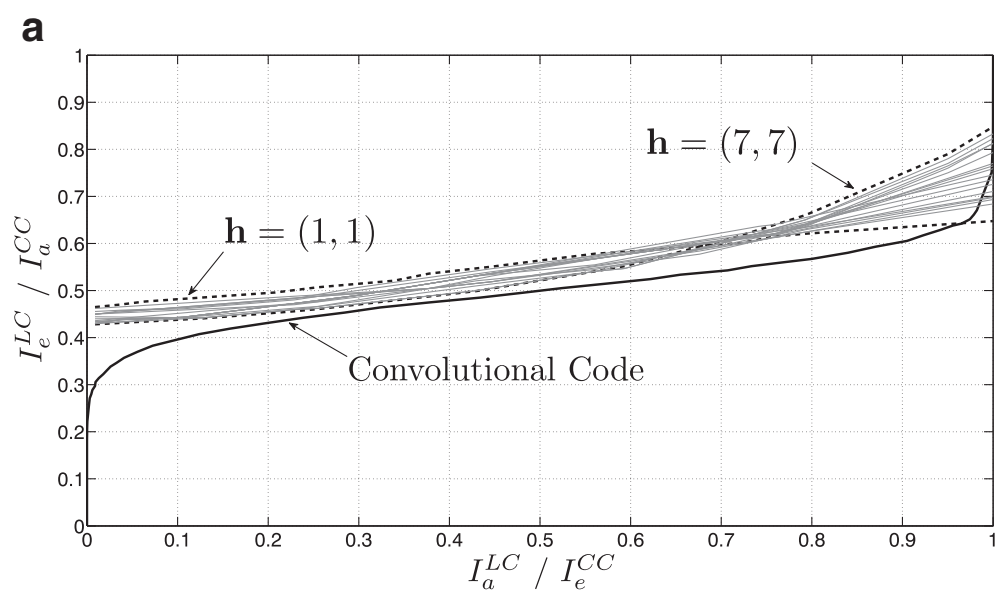

b

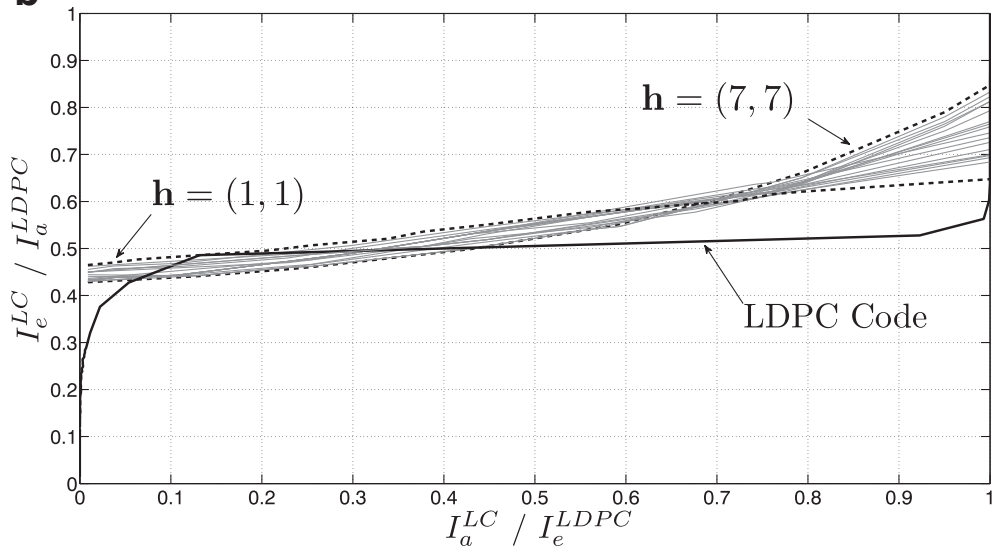

Figure 5 EXIT charts of the LC decoder. Different network coefficients along with our scheme (a) and the LDPC code from [4] (b).

signal-to-noise ratio (SNR). Hence, to open the gap for the LDPC case a larger SNR will be required.

In light of the above analysis, it is expected that our scheme will outperform the scheme in [4] which is based on codeword lengths two orders of magnitude higher. This assumption is corroborated by the simulation in the next section.

\section{Results}

In order to assess the performance of the proposed code, several Monte Carlo simulations have been run using a 4state $[5,7]_{8}$ non-systematic rate- $1 / 2$ convolutional code ${ }^{2}$. The source generates $K=13$ information bits, and a zero-bit tail is appended at the source sequence in order to properly terminate the convolutional code. Specifically, we add 3 zeros so the block entering the convolutional code has 16 bits, and hence, the encoded sequence has $N=32$ binary symbols. We have considered a 16-QAM constellation (i.e., $q=4$ and $M=8$ complex dimensions) and $\mu$ to be the Gray mapping. Moreover, $\delta$ has been set to 4 and the interleaver $\Pi(\cdot)$ has been randomly generated with a spread factor of $q=4$. Finally, the number of iterations completed by the decoding algorithm at the relays is set 100 .

The number of nodes has been set to $|\mathfrak{M}|=5$. That is, the source transmits its message to the destination with the aid of three intermediate nodes. Therefore, a total of 20 baseband symbols (i.e., complex dimensions) are transmitted through the system leading to a spectral efficiency of $\rho_{S}=13 / 20$ (information bits per complex dimension). Finally, the vector coefficients are chosen as $\{(1,0),(0,1),(1,1),(2,1),(1,2)\}$, and, for the sake of simplicity, we have assumed equal output power at each of the compounding nodes of the network.

The first set of simulations aims at analyzing the performance of the DF scheme at the different relay nodes and therefore, at different distances from the source. Figure 6 plots the bit error rate (BER) versus $E_{b} / N_{0}$ at nodes $j=$ $1,2,3,4$. Notice that when $j=k$, the number of relay nodes considered in the network is $k-1$ and node $k$ becomes in this case the destination node. Thus, the distance between the source and the destination node is $k$ units. Following the comparison carried out in Section 4, 


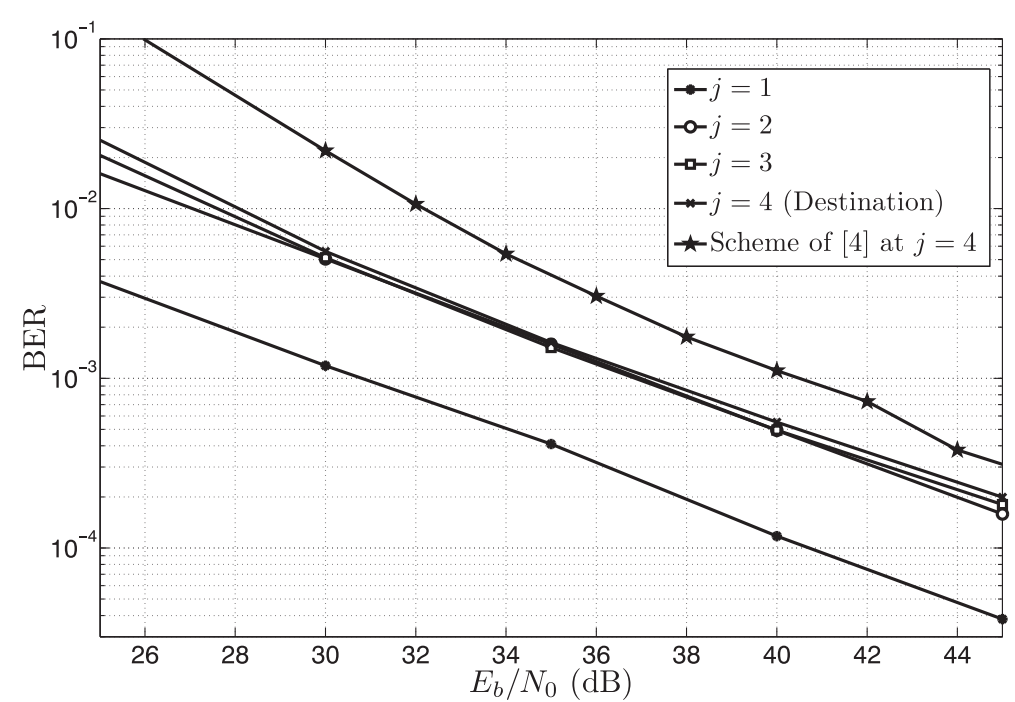

Figure 6 BER performance of the proposed scheme at all the nodes for 100 iterations. Also plotted the performance of [4] at the destination.

the figure also shows the BER performance of the scheme proposed in [4] at the destination $(j=4)$.

It can be observed that, after the first hop $(j=1)$, the performance at high values of SNR for the remaining nodes $(j=2,3,4)$ is almost the same. In fact, for SNR $=30$ to $40 \mathrm{~dB}$, the BER curves for $j=2,3$, 4 even overlap. The main reason for this behavior is the following: On the one hand, for small number of relays, the increment of diversity is not significant due to the pathloss attenuation suffered by the signals. On the other hand, as the number of relays increases, the achieved diversity starts to increase since the pathloss attenuation becomes less significant due to the increment of incoming signals. Hence, adding more relays in order to reach a distant point practically does not degrade the performance of the system, which makes the proposed scheme suitable for multi-hop wireless networks. This advantage becomes more evident in Figure 7 where the proposed scheme is tested over a larger network with 10 nodes $(|\mathfrak{M}|=$ 10). The performance of this larger network remains practically constant when the SNR is sufficiently high (SNR $\geq 30 \mathrm{~dB}$ ).

It can also be said that the proposed DF scheme outperforms the schemes presented in [4] in more than $3 \mathrm{~dB}$ (and consequently [5] and in [6]). This fact confirms the insight given by the previous EXIT charts study. Furthermore, it is important to remark that, for the same spectral efficiency, the proposed scheme uses a total of 20 complex dimensions in comparison to the 4,500 used in the referenced scheme.

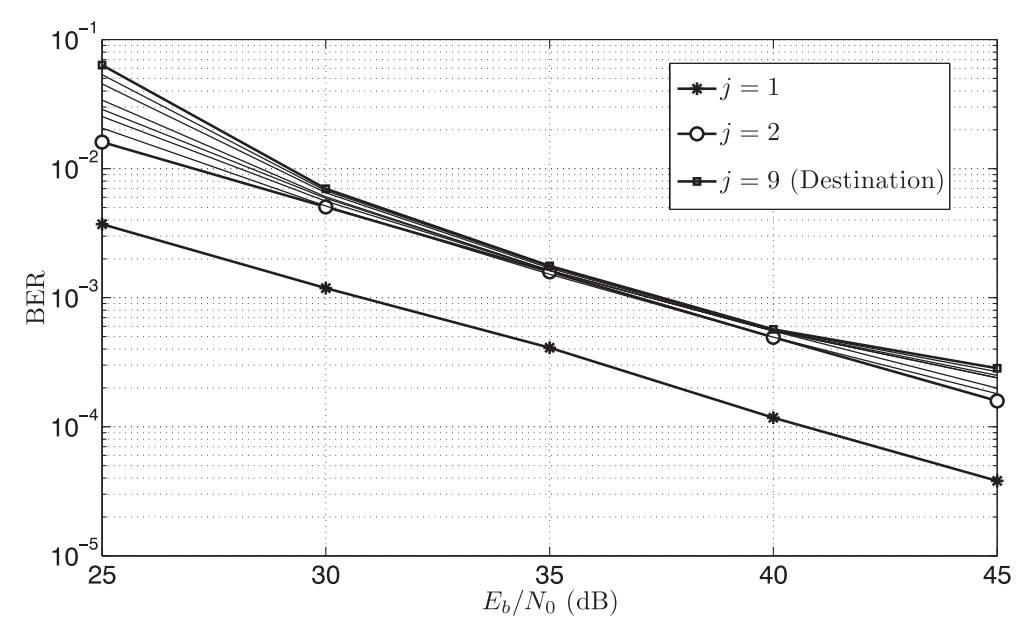

Figure 7 BER performance of the proposed scheme at all the nodes. The network is composed of 10 nodes instead of $5(|\mathfrak{M}|=10)$ 

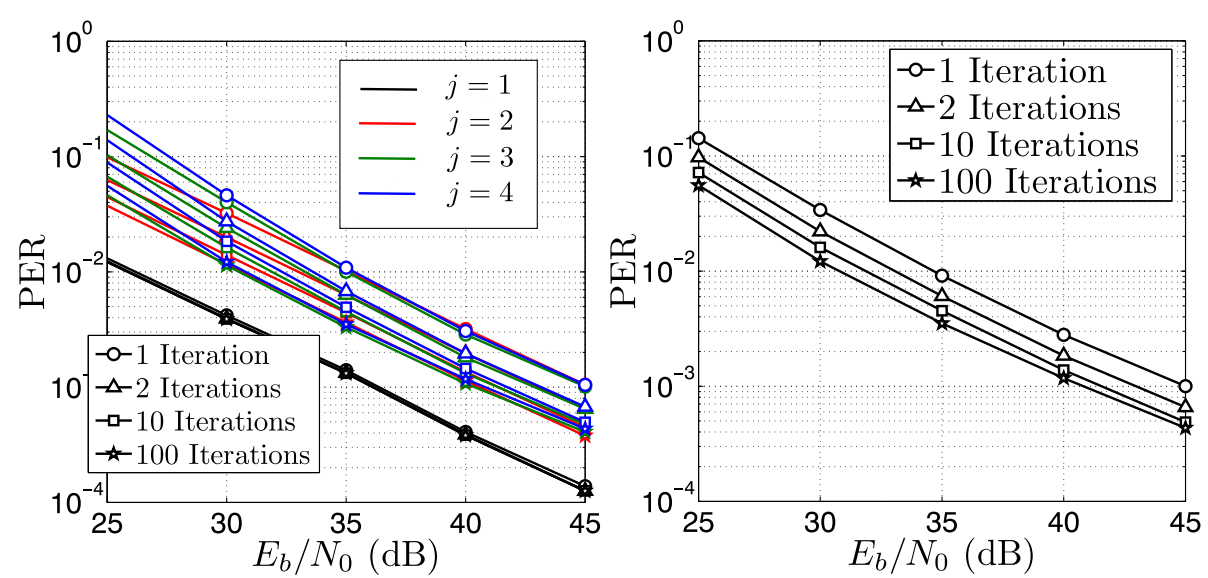

Figure 8 Performance of the proposed scheme in terms of PER for different amount of iterations at the relays and 100 iterations at the destination. At every receiving node of the network $(j=1,2,3,4)$ on the left and only for the destination $(j=4)$ on the right.

Another interesting application of the proposed scheme is networks in which the relays share their resources with other networks. In this case, the number of iterations at the relays should be kept small to reduce their computational load. However, a performance degradation is expected for choosing a low number of iteration. To check the performance of this scheme, a different set of simulations has been studied. This is shown in Figure 8 which plots the packet-error rate (PER) versus $E_{b} / N_{0}$ at the destination where we fix the number of iterations at the destination in 100, and vary the iterations performed at the relays with values from the set $\{1,2,10,100\}$. As previously, the number of nodes of the network has been set to $|\mathfrak{M}|=5$.

Figure 8 (left) shows that at the first relay $(j=1)$ there is a small improvement in the performance by increasing the number of iterations. This situation changes completely for the remaining nodes where there is approximately a $4 \mathrm{~dB}$ gap between the performance for 1 iteration and 100 iterations. Perhaps, it is more insightful to focus on the performance at the destination $(j=4)$, plotted in Figure 8 (right). In this figure, we see that when the number of iterations at the relay is set to 2,10 , and 100 , gains of $2 \mathrm{~dB}$, $3.5 \mathrm{~dB}$, and $4 \mathrm{~dB}$ are obtained, respectively, with respect to a single iteration. Notice also that going from 10 iterations to 100 iterations conveys a gain of only $0.5 \mathrm{~dB}$; hence, the gain obtained for performing more than 10 iterations is negligible, which makes the scheme suitable for relays with shared resources.

\section{Conclusion}

We have presented a decode-and-forward scheme for multihop wireless networks with orthogonal channels. The proposed scheme blends together convolutional channel coding with linear combination of blocks of data over a finite field. According to EXIT chart analysis and simulation results, the proposed DF scheme clearly outperforms previously schemes in the literature by using only a total of 20 complex dimensions. This latter fact makes the proposed scheme particularly attractive for low-latency applications. Furthermore, it is also suitable for large multihop networks as a negligible performance degradation is obtained when adding more hops. Finally, this scheme is suitable for relays with shared resources since most of the iterative gain at the decoding is achieved in less than 10 iterations of the decoding process.

\section{Competing interests}

The authors declare that they have no competing interests.

\section{Acknowledgements}

This work was supported in part by the Spanish Ministry of Science \& Innovation through the COMONSENS (CSD200800010) and COSIMA (TEC2010-19545-C04-02) projects, as well as by Cátedra Telefónica at University of Navarra.

Received: 10 October 2014 Accepted: 26 April 2015

Published online: 13 May 2015

\section{References}

1. R Babaee, NC Beaulieu, Cross-layer design for multihop wireless relaying networks. IEEE Trans. Wireless Comm. 9, 3522-3531 (2010)

2. E Morgado, I Mora-Jimenez, JJ Vinagre, J Ramos, AJ Caamano, End-to-end average ber in multihop wireless networks over fading channels. IEEE Trans. Wireless Comm. 9, 2478-2487 (2010)

3. R Babaee, NC Beaulieu, Exact evaluation of ergodic capacity for multihop variable-gain relay networks: a unified framework for generalized fading channels. IEEE Trans. Vehicular Tech. 59, 4181-4186 (2010)

4. M Hernaez, PM Crespo, in Proceedings of VTC-Spring 11. A novel scheme for message-forwarding in multi-hop ad-hoc wireless networks (IEEE, Budapest, Hungary, 2011)

5. $\mathrm{X}$ Bao, $\mathrm{J} \mathrm{Li}$, in Proceedings of SECON. Progressive network coding for message-forwarding in ad-hoc wireless networks (IEEE, Reston, USA, 2006)

6. C Hausl, F Schreckenbach, I Oikonomidis, G Bauchi, in Proceedings of Allerton, University of Illinois, USA. Iterative network and channel decoding on a tanner graph, (2005)

7. FR Kschischang, BJ Frey, HA Loeliger, Factor graphs and the sum-product algorithm. IEEE Trans. Inf. Theory. 44, 498-519 (2001) 
8. M Hernaez, I Alustiza, PM Crespo, JD Ser, in Proceedings of VTC-Fall13. A decode-and-forward scheme for multihop wireless networks (IEEE Las Vegas, USA, 2013)

9. J Hagenauer, in Proceedings of EUSIPCO. The exit chart: Introduction to extrinsic information transfer in iterative processing (EURASIP Vienna, Austria, 2004)

10. M Hernaez, PM Crespo, JD Ser, Flexible channel coding approach for short-length codewords. IEEE Comm. Lett. 16(9), 1508-1511 (2012)

11. JW Lee, RE Blahut, Lower bound on ber of finite-length turbo codes based on exit characteristics. IEEE Comm. Lett. 8(4), 238-240 (2004)

12. M Hernaez, PM Crespo, JD Ser, On the design of a novel joint network-channel coding scheme for multiple access relay channels. IEEE J. Sel. Areas Commun. 31(8), 1368-1378 (2013)

13. F Brannstrom, LK Rasmussen, AJ Grant, Convergence analysis and optimal scheduling for multiple concatenated codes. IEEE Trans. Inf. Theory. 51, 3354-3364 (2005)

\section{Submit your manuscript to a SpringerOpen ${ }^{\mathcal{O}}$ journal and benefit from:}

- Convenient online submission

- Rigorous peer review

- Immediate publication on acceptance

- Open access: articles freely available online

- High visibility within the field

- Retaining the copyright to your article

Submit your next manuscript at $\gg$ springeropen.com 